\title{
Dynamic Output-Feedback Passivity Control for Fuzzy Systems under Variable Sampling
}

\author{
Hongyi Li, ${ }^{1,2}$ Xingjian Sun, ${ }^{2}$ H. R. Karimi, ${ }^{3}$ and Ben Niu ${ }^{2}$ \\ ${ }^{1}$ College of Engineering, Bohai University, Jinzhou, Liaoning 121013, China \\ ${ }^{2}$ School of Mathematics and Physics, Bohai University, Jinzhou, Liaoning 121013, China \\ ${ }^{3}$ Department of Engineering, Faculty of Engineering and Science, University of Agder, Grimstad, Norway
}

Correspondence should be addressed to Hongyi Li; lihongyi2009@gmail.com

Received 27 June 2013; Accepted 22 August 2013

Academic Editor: Xudong Zhao

Copyright (C) 2013 Hongyi Li et al. This is an open access article distributed under the Creative Commons Attribution License, which permits unrestricted use, distribution, and reproduction in any medium, provided the original work is properly cited.

\begin{abstract}
This paper concerns the problem of dynamic output-feedback control for a class of nonlinear systems with nonuniform uncertain sampling via Takagi-Sugeno (T-S) fuzzy control approach. The sampling is not required to be periodic, and the state variables are not required to be measurable. A new type fuzzy dynamic output-feedback sampled-data controller is constructed, and a novel time-dependent Lyapunov-Krasovskii functional is chosen for fuzzy systems under variable sampling. By using Lyapunov stability theory, a sufficient condition for very-strict passive analysis of fuzzy systems with nonuniform uncertain sampling is derived. Based on this condition, a novel fuzzy dynamic output-feedback controller is designed such that the closed-loop system is very-strictly passive. The existence condition of the controller can be solved by convex optimization approach. Finally, a numerical example is provided to demonstrate the effectiveness of the proposed method.
\end{abstract}

\section{Introduction}

The fuzzy logic control [1-5] is one of the most effective approaches to handle complex nonlinear systems and has been applied into various real systems. Takagi-Sugeno (TS) [6] fuzzy model is a popular and effective method to represent complex nonlinear systems into a weighted sum of some simple linear subsystems [7-9]. Complex nonlinear systems can be represented by T-S fuzzy model in a set of IF-THEN rules [10]. Recently, many stability and control problems were investigated for T-S fuzzy systems; see, for example, [11-15]. The authors in [10] proposed fuzzy control systems design and analysis results via linear matrix inequality (LMI) approach, and paper [7] presented a survey on recent advances and the state of the art of analysis and design of model based fuzzy control systems. More recently, many results on stability analysis, controller synthesis, and filter design for T-S fuzzy systems with time delays have been reported in [16-24] and the references therein.

On the other hand, it is significant to study the sampleddata control problems for practical control systems [25-31].
In the past few decades, there are two main approaches being utilized to solve stability analysis and control synthesis problems for sampled-data systems. The first one is that a sampled-data system is structured as a discrete-time system [29]. Another is to structure a sampled-data system as a continuous-time system with a delayed control input $[31,32]$. It has been shown that the first method is more difficult to analyze or synthesize for complex systems than the second one. Recently, the sampled-data control problem for T-S fuzzy systems via input delay method has received considerable attention. Some state-feedback control design methods have been proposed [32-35], and observer-based control approach has been used in [36]. Via input delay approach, the stabilization of nonuniform sampling fuzzy control systems has been studied in [34]. However, it should be mentioned that there are few results on fuzzy dynamic output-feedback controller design for T-S fuzzy systems with nonuniform uncertain sampling. More recently, the passivity analysis and passive control problems for fuzzy systems have received considerable attention, and many results have been reported [37-39]. In [39], the authors considered very-strict 
passive control for T-S fuzzy systems with both state and input delays. When the state variables are not measurable, there are few results about output-feedback control for T-S fuzzy systems based on passive theory, which motivates this study.

In this paper, a new type of dynamic output-feedback control is designed for a class of nonlinear systems with variable sampling. Firstly, by choosing a novel time-dependent Lyapunov-Krasovskii functional and using Lyapunov stability theory, a sufficient condition is presented for very-strict passive analysis for fuzzy systems with nonuniform uncertain sampling. Based on the conception of very-strict passivity, a new sampled-data dynamic output-feedback controller is designed to guarantee that the closed-loop system is very strictly passive. The existence condition of the controller can be solved by convex optimization approach. Finally, a numerical example is given to show the effectiveness of the proposed results. The main contributions of this paper can be summarized as follows: (i) a new dynamic output-feedback sampled-data controller is constructed for T-S fuzzy system with variable sampling; (ii) a novel time-dependent and fuzzy membership dependent Lyapunov-Krasovskii functional is chosen for synthesizing output-feedback sampled-data controller for T-S fuzzy system. The remainder of the paper is organized as follows. The problem to be addressed is formulated in Section 2, and dynamic output-feedback controller is designed for fuzzy systems with variable sampling in Section 3. A numerical example is provided in Section 4 to demonstrate the effectiveness of the developed approach, and Section 5 concludes the paper.

Notation. $\mathscr{R}^{n}$ stands for the $n$-dimensional Euclidean space. $I$ and 0 represent, respectively, identity matrix and zero matrix, and $[A]_{s}$ is used to denote $A+A^{T}$ for simplicity. $\operatorname{diag}\{\cdots\}$ stands for a block-diagonal matrix. $P>0(\geq 0)$ stands for a symmetric and positive definite (semidefinite), and the superscript " $T$ " and "- 1 " stand for matrix transposition and inverse. In symmetric block matrices, we use an asterisk $(\star)$ to represent a term that is induced by symmetry.

\section{Problem Formulation}

Consider the following T-S fuzzy systems.

Plant Rule i. IF $\theta_{1}(t)$ is $N_{i 1}$ and $\cdots$ and $\theta_{p}(t)$ is $N_{i p}$ THEN

$$
\begin{aligned}
& \dot{x}(t)=A_{i} x(t)+B_{i} u(t)+B_{w i} w(t), \\
& z(t)=C_{i} x(t)+D_{i} u(t)+D_{w i} w(t), \\
& y(t)=C_{y i} x(t), \quad i=1,2,3, \ldots, r,
\end{aligned}
$$

where $x(t) \in \mathscr{R}^{n}$ is the state vector, $u(t) \in \mathscr{R}^{m}$ is the control input, $w(t) \in \mathscr{R}^{p}$ is the disturbance input, $z(t) \in \mathscr{R}^{q}$ is the control output, and $y(t) \in \mathscr{R}^{s}$ is the measured output. $A_{i}, B_{i}$, $B_{w i}, C_{i}, D_{i}, D_{w i}$, and $C_{y i}$ are system matrices with appropriate dimensions. The scalar $r$ is the number of IF-THEN rules. $\theta_{1}(t), \theta_{1}(t), \ldots, \theta_{p}(t)$ are the premise variables, $N_{i j}$ is the fuzzy set, $i=1,2,3, \ldots, r, j=1,2,3, \ldots, p$. For a given input and output $(x(t), u(t))$, the defuzzified output of system (1) is inferred as follows:

$$
\begin{aligned}
& \dot{x}(t)=\sum_{i=1}^{r} h(\theta(t))\left[A_{i} x(t)+B_{i} u(t)+B_{w i} w(t)\right], \\
& z(t)=\sum_{i=1}^{r} h(\theta(t))\left[C_{i} x(t)+D_{i} u(t)+D_{w i} w(t)\right], \\
& y(t)=\sum_{i=1}^{r} h(\theta(t)) C_{y i} x(t),
\end{aligned}
$$

where $h_{i}(\theta(t))$ denotes the normalized membership functions satisfying

$$
h_{i}(\theta(t))=\frac{\omega_{i}(\theta(t))}{\sum_{i=1}^{r} \omega_{i}(\theta(t))}, \quad \omega_{i}(\theta(t))=\prod_{j=1}^{r} N_{i j}\left(\theta_{j}(t)\right),
$$

where $N_{i j}\left(\theta_{j}(t)\right)$ is the grade of membership of $\theta_{j}(t)$ in $N_{i j}$. Notice the facts that $\omega_{i}(\theta(t)) \geq 0$ and $\sum_{i=1}^{r} \omega_{i}(\theta(t))>0, \forall t \geq$ 0 . Then, it can be seen that $h_{i}(\theta(t)) \geq 0$ and $\sum_{i=1}^{r} h_{i}(\theta(t))=$ 1 for $i=1,2, \ldots, r$. Suppose that the updating signal is successfully transmitted from the sampler to the controller and to the zero-order-hold $(\mathrm{ZOH})$ at the instant $t_{k}$. It is assumed that the sampling intervals are bounded:

$$
t_{k+1}-t_{k} \leq h
$$

Here $h$ denotes the maximum time span between the time $t_{k}$ at which the state is sampled and the time $t_{k+1}$ at which the next update arrives at the destination. The initial conditions of $x(t)$ and $u(t)$ are given as $x(t)=\varphi(t)$ and $u(t)=0$ for $t \in\left[t_{0}-h, t_{0}\right]$, where $\varphi(t)$ is a differentiable function. When the state variables are unmeasurable or unknown, the state-feedback control method is not available. In this paper, a novel dynamic output-feedback sampled-date controller is constructed as follows.

Controller Rule i. IF $\theta_{j}\left(t_{k}\right)$ is $N_{j 1}$ and $\cdots \theta_{p}\left(t_{k}\right)$ is $N_{j p}$ THEN

$$
\begin{aligned}
& \dot{\hat{x}}(t)=A_{c j} \hat{x}(t)+A_{d j} \hat{x}\left(t_{k}\right)+B_{c j} y\left(t_{k}\right), \\
& u(t)=C_{c j} \widehat{x}(t) .
\end{aligned}
$$

Similar to the fuzzy model, the same fuzzy rule is used to construct the following overall fuzzy control law:

$$
\begin{aligned}
& \dot{\hat{x}}(t)=\sum_{j=1}^{r} h_{j}\left(\theta\left(t_{k}\right)\right)\left[A_{c j} \hat{x}(t)+A_{d j} \hat{x}\left(t_{k}\right)+B_{c j} y\left(t_{k}\right)\right], \\
& u(t)=\sum_{j=1}^{r}\left(\theta\left(t_{k}\right)\right) C_{c j} \hat{x}(t),
\end{aligned}
$$

where $\widehat{x} \in \mathscr{R}^{n}$ is the state vector of the dynamic controller and $t_{k}(k=0,1,2, \ldots)$ denotes the $k$ th sampling instant, $t_{0} \geq 0$, and $\lim _{k \rightarrow \infty} t_{k}=\infty . A_{c j}, A_{c d j}, B_{c j}$, and $C_{c j}(j=1,2, \ldots, r)$ are control matrices with appropriate dimensions. $t_{k+1}$ is 
the updating instant time of the ZOH after $t_{k}$. Denote $d(t)=$ $t-t_{k}$ for $t_{k} \leq t \leq t_{k+1}$. It is clear that $0 \leq d(t) \leq t_{k+1}-t_{k} \leq h$. It can be seen that $d(t)$ is sawtooth structure, that is, piecewiselinear with derivative $\dot{d}(t)=1, t \neq t(k)$. Utilizing $d(t)=t-t_{k}$, the following systems can be obtained:

$$
\begin{aligned}
\dot{\hat{x}}(t)= & \sum_{j=1}^{r} h_{j}(\theta(t-d(t))) \\
& \quad \times\left[A_{c j} \widehat{x}(t)+A_{d j} \widehat{x}(t-d(t))+B_{c j} y(t-d(t))\right], \\
u(t)= & \sum_{j=1}^{r} h_{j}(\theta(t-d(t))) C_{c j} \widehat{x}(t) .
\end{aligned}
$$

Applying the fuzzy controller (7) to system (2) and yielding the closed-loop system as follows:

$$
\begin{gathered}
\dot{\bar{x}}(t)=\sum_{i=1}^{r} \sum_{j=1}^{r} h_{i} h_{j}^{k}\left[\bar{A}_{i j} \bar{x}(t)+\bar{B}_{i j} \bar{x}(t-d(t))+\bar{B}_{w i j} w(t)\right], \\
z(t)=\sum_{i=1}^{r} \sum_{j=1}^{r} h_{i} h_{j}^{k}\left[\bar{C}_{i j} \bar{x}(t)+\bar{D}_{i j} w(t)\right],
\end{gathered}
$$

where

$$
\begin{array}{cc}
\bar{A}_{i j}=\left[\begin{array}{cc}
A_{i} & B_{i} C_{c j} \\
0 & A_{c j}
\end{array}\right], \quad \bar{B}_{i j}=\left[\begin{array}{cc}
0 & 0 \\
B_{c j} C_{y i} & A_{c d j}
\end{array}\right], \\
\bar{B}_{w i j}=\left[\begin{array}{c}
B_{w i} \\
0
\end{array}\right], \quad \bar{C}_{i j}=\left[\begin{array}{ll}
C_{i} & D_{i} C_{c j}
\end{array}\right], \\
\bar{D}_{w i j}=D_{w i}, \quad \bar{x}(t)=\left[\begin{array}{l}
x(t) \\
\widehat{x}(t)
\end{array}\right], \\
\sum_{i=1}^{r} h_{i}=\sum_{i=1}^{r} h_{i}(\theta(t)), \quad \sum_{j=1}^{r} h_{j}^{k}=\sum_{j=1}^{r} h_{j}(\theta(t-d(t))) .
\end{array}
$$

\section{Main Results}

In order to develop the main results, the definition is introduced as follows.

Definition 1 (see [39]). System (8) is said to be very-strictly passive if there exist constants $\varepsilon>0, \delta>0$ and $\rho$ such that

$$
\begin{aligned}
& 2 \int_{0}^{t} z^{T}(s) w(s) d s \\
& \quad \geq \rho+\varepsilon \int_{0}^{t} z^{T}(s) z(s) d s+\delta \int_{0}^{t} w^{T}(s) w(s) d s
\end{aligned}
$$

holds for all $t \geq 0$.

In this section, a novel dynamic output-feedback sampled-data controller for system in (2) is designed to guarantee that the closed-loop system (8) is very-strictly passive. For given dynamic output-feedback control gain matrices $\bar{A}_{i j}, \bar{B}_{i j}, \bar{B}_{w i j}, \bar{C}_{i j}$, and $\bar{D}_{w i j}$, the condition of passivity analysis is proposed for the closed-loop system (8).

Theorem 2. Consider the closed-loop system (8), for given scalar $h>0$ and matrices with appropriate dimensions $\bar{A}_{i j}$, $\bar{B}_{i j}, \bar{B}_{w i j}, \bar{C}_{i j}$, and $\bar{D}_{w i j}$; the closed-loop system (8) is verystrictly passive, if there exist scalars $\varepsilon>0, \delta>0$ and matrices $P=P^{T}>0, L_{i j}=L_{i j}^{T}>0, Q_{i j}=Q_{i j}^{T}>0, N_{1}=N_{1}^{T}>0$, $Z_{1}=Z_{1}^{T}>0$, and $Z_{2}=Z_{2}^{T}>0$ with appropriate dimensions, such that the following LMIs hold for $i, j=1,2, \ldots, r$ :

$$
\begin{aligned}
& {\left[\begin{array}{ccccc}
\Phi_{11 i j}+\Theta_{1 i j} & \Phi_{12 i j} & \Phi_{13 i j} & \Phi_{14 i j} & \Phi_{15 i j} \\
\star & -\varepsilon I & 0 & 0 & 0 \\
\star & \star & -L_{i j} & 0 & 0 \\
\star & \star & \star & -\varepsilon I & 0 \\
\star & \star & \star & \star & \Phi_{55 i j}
\end{array}\right]<0,} \\
& {\left[\begin{array}{ccc}
\Phi_{11 i j}+\Theta_{2 i j} & \Phi_{12 i j} & \Phi_{13 i j} \\
\star & -\varepsilon I & 0 \\
\star & \star & -L_{i j}
\end{array}\right]<0,} \\
& Q_{i j}<L_{j i},
\end{aligned}
$$

where

$$
\begin{gathered}
\Phi_{11 i j}=\left[\begin{array}{cccc}
F_{11 i j} & F_{12 i j} & 0 & F_{14 i j} \\
\star & F_{22 i j} & Q_{i j} & 0 \\
\star & \star & F_{33 i j} & 0 \\
\star & \star & \star & F_{44 i j}
\end{array}\right], \\
\Theta_{1 i j}=\left[\begin{array}{cccc}
-Q_{i j} & Q_{i j} & 0 & 0 \\
Q_{i j} & -Q_{i j} & 0 & 0 \\
0 & 0 & 0 & 0 \\
0 & 0 & 0 & 0
\end{array}\right]
\end{gathered}
$$$$
\Theta_{2 i j}=\left[\begin{array}{cccc}
0 & 0 & 0 & 0 \\
0 & -Q_{i j} & Q_{i j} & 0 \\
0 & Q_{i j} & -Q_{i j} & 0 \\
0 & 0 & 0 & 0
\end{array}\right] \text {, }
$$

$$
\begin{gathered}
\Phi_{15 i j}=\left[\begin{array}{cccc}
h \bar{A}_{i j} & h \bar{B}_{i j} & 0 & h \bar{B}_{w i j} \\
h Z_{1} \bar{A}_{i j} & h Z_{1} \bar{B}_{i j} & 0 & h Z_{1} \bar{B}_{w i j}
\end{array}\right]^{T}, \\
F_{11 i j}=P \bar{A}_{i j}+\bar{A}_{i j}^{T} P-Q_{i j}-\frac{1}{h} Z_{1}-Z_{2}+N_{1},
\end{gathered}
$$

$$
F_{12 i j}=P \bar{B}_{i j}+Q_{i j}+\frac{1}{h} Z_{1}+Z_{2}
$$

$$
\begin{gathered}
F_{14 i j}=P \bar{B}_{w i j}-\bar{C}_{i j}^{T}, \quad F_{22 i j}=-2 Q_{i j}-\frac{1}{h} Z_{1}-Z_{2}, \\
F_{33 i j}=-Q_{i j}-N_{1}, \quad F_{44 i j}=\delta I-\bar{D}_{w i j}-\bar{D}_{w i j}^{T}, \\
\Phi_{12 i j}=\left[\begin{array}{llll}
\bar{C}_{i j} & 0 & 0 & \bar{D}_{w i j}
\end{array}\right]^{T}, \\
\Phi_{13 i j}=\left[\begin{array}{lllll}
h R_{i j} \bar{A}_{i j} & h R_{i j} \bar{B}_{i j} & 0 & h R_{i j} \bar{B}_{w i j}
\end{array}\right]^{T},
\end{gathered}
$$




$$
\begin{gathered}
\Phi_{14 i j}=\left[\begin{array}{llll}
h Z_{2} & -h Z_{2} & 0 & 0
\end{array}\right]^{T}, \\
\Phi_{55 i j}=\operatorname{diag}\left\{-h I,-h Z_{1}\right\} .
\end{gathered}
$$

Proof. Consider the following Lyapunov-Krasovskii functional:

$$
\begin{gathered}
V(t)=V_{1}(t)+V_{2}(t)+V_{3}(t) \\
V_{1}(t)=\bar{x}^{T}(t) P \bar{x}(t)+\int_{t-h}^{t} \bar{x}^{T}(s) N_{1} \bar{x}(s) d s \\
V_{2}(t)=h \int_{-h}^{0} \int_{t+\theta}^{t} \dot{\bar{x}}^{T}(s) L_{i j} \dot{\bar{x}}(s) d s d \theta
\end{gathered}
$$

$$
\begin{aligned}
& V_{3}(t) \\
& =(h-d(t)) \\
& \quad \times \int_{t-d(t)}^{t} \dot{\bar{x}}^{T}(s) Z_{1} \dot{\bar{x}}(s) d s+(h-d(t)) \vartheta^{T}(t) Z_{2} \vartheta(t),
\end{aligned}
$$

where $L(t)=\sum_{i=1}^{r} \sum_{j=1}^{r} h_{i} h_{j}^{k} L_{i j}>0$ and $\vartheta=(x(t)-x(t-d(t)))$, $t_{k} \leq t \leq t_{k+1}$. Hence $V(t)>0$ and is continuous in time. The derivatives of $V_{1}(t), V_{2}(t)$, and $V_{3}(t)$ with time $t$ can be obtained as

$$
\begin{gathered}
\dot{V}_{1}(t)=2 \bar{x}^{T} P \dot{\bar{x}}(t)+\bar{x}^{T}(t) N_{1} \bar{x}(t)-\bar{x}^{T}(t-h) N_{1} \bar{x}(t-h), \\
\dot{V}_{2}(t)=h^{2} \dot{\bar{x}}^{T}(t) L(t) \dot{\bar{x}}(t)-h \int_{t-h}^{t} \dot{\bar{x}}^{T}(s) L(s) \dot{\bar{x}}(s) d s \\
\dot{V}_{3}(t)=-\int_{t-d(t)}^{t} \dot{\bar{x}}^{T}(s) Z_{1} \dot{\bar{x}}(s) d s+(h-d(t)) \dot{\bar{x}}^{T}(t) Z_{1} \dot{\bar{x}}(t) \\
-\vartheta^{T}(t) Z_{2} \vartheta(t)+2(h-d(t)) \vartheta^{T}(t) Z_{2} \dot{\bar{x}}(t) .
\end{gathered}
$$

It can be seen from condition (13) that $Q(t)=$ $\sum_{i=1}^{r} \sum_{j=1}^{r} h_{i} h_{j}^{k} Q_{i j}<L(t)$. For the second term in $\dot{V}_{2}(t)$ the following inequalities can be obtained:

$$
\begin{aligned}
-h \int_{t-h}^{t} & \dot{\bar{x}}^{T}(s) L(s) \dot{\bar{x}}(s) d s \\
< & -h \int_{t-h}^{t} \dot{\bar{x}}^{T}(s) Q(s) \dot{\bar{x}}(s) d s \\
= & -h \int_{t-d(t)} \dot{\bar{x}}^{T}(s) Q(s) \dot{\bar{x}}(s) d s \\
& -h \int_{t-h}^{t-d(t)} \dot{\bar{x}}^{T}(s) Q(s) \dot{\bar{x}}(s) d s \\
= & -(h-d(t)) \int_{t-d(t)}^{t} \dot{\bar{x}}^{T}(s) Q(s) \dot{\bar{x}}(s) d s \\
& -d(t) \int_{t-d(t)}^{t} \dot{\bar{x}}^{T}(s) Q(s) \dot{\bar{x}}(s) d s \\
& -(h-d(t)) \int_{t-h}^{t-d(t)} \dot{\bar{x}}^{T}(s) Q(s) \dot{\bar{x}}(s) d s \\
& -d(t) \int_{t-h}^{t-d(t)} \dot{\bar{x}}^{T}(s) Q(s) \dot{\bar{x}}(s) d s
\end{aligned}
$$

$$
\begin{aligned}
\leq & -\frac{h-d(t)}{h} \xi_{1}^{T}(t) Q(t) \xi_{1}(t)-\xi_{1}^{T}(t) Q(t) \xi_{1}(t) \\
& -\xi_{2}^{T}(t) Q(t) \xi_{2}(t)-\frac{d(t)}{h} \xi_{2}^{T}(t) Q(t) \xi_{2}(t) \\
= & \xi_{3}^{T}(t) U_{1} \xi_{3}(t)+\frac{h-d(t)}{h} \xi_{3}^{T}(t) U_{2} \xi_{3}(t) \\
& +\frac{d(t)}{h} \xi_{3}^{T}(t) U_{3} \xi_{3}(t),
\end{aligned}
$$

where

$$
\begin{aligned}
& \xi_{1}(t)=\int_{t-d(t)}^{t} \dot{\bar{x}}(s) d s, \quad \xi_{2}(t)=\int_{t-h}^{t-d(t)} \dot{\bar{x}}(s) d s, \\
& \xi_{3}^{T}(t)=\left[\begin{array}{lll}
x^{T}(t) & x^{T}(t-d(t)) & x^{T}(t-h)
\end{array}\right], \\
& U_{1}=\left[\begin{array}{ccc}
-Q(t) & Q(t) & 0 \\
Q(t) & -2 Q(t) & Q(t) \\
0 & Q(t) & -Q(t)
\end{array}\right], \\
& U_{2}=\left[\begin{array}{ccc}
-Q(t) & Q(t) & 0 \\
Q(t) & -Q(t) & 0 \\
0 & 0 & 0
\end{array}\right] \text {, } \\
& U_{3}=\left[\begin{array}{ccc}
0 & 0 & 0 \\
0 & -Q(t) & Q(t) \\
0 & Q(t) & -Q(t)
\end{array}\right] \text {. }
\end{aligned}
$$

Similarly, for the first term in $\dot{V}_{3}(t)$, we can have

$$
\begin{aligned}
& -\int_{t-d(t)}^{t} \dot{\bar{x}}^{T}(s) Z_{1} \dot{\bar{x}}(s) d s \\
& \quad \leq\left[\begin{array}{c}
\bar{x}(t) \\
\bar{x}(t-d(t))
\end{array}\right]^{T}\left[\begin{array}{cc}
-\frac{1}{h} Z_{1} & \frac{1}{h} Z_{1} \\
\frac{1}{h} Z_{1} & -\frac{1}{h} Z_{1}
\end{array}\right]\left[\begin{array}{c}
\bar{x}(t) \\
\bar{x}(t-d(t))
\end{array}\right] .
\end{aligned}
$$

For the last term in $\dot{V}_{3}(t)$, one can have

$$
\begin{aligned}
2(h- & d(t)) \vartheta^{T}(t) Z_{2} \dot{\bar{x}}(t) \\
& \leq \frac{h-d(t)}{h}\left[h \vartheta^{T}(t) Z_{2} Z_{2} \vartheta(t)+h \dot{\bar{x}}^{T}(t) \dot{\bar{x}}(t)\right] .
\end{aligned}
$$

In addition, the following inequality holds:

$$
\begin{aligned}
z^{T}(t) z(t)= & \left\{\sum_{i=1}^{r} \sum_{j=1}^{r} h_{i} h_{j}^{k}\left[\bar{C}_{i j} \bar{x}(t)+\bar{D}_{i j} w(t)\right]\right\}^{T} \\
& \times\left\{\sum_{i=1}^{r} \sum_{j=1}^{r} h_{i} h_{j}^{k}\left[\bar{C}_{i j} \bar{x}(t)+\bar{D}_{i j} w(t)\right]\right\}
\end{aligned}
$$




$$
\begin{gathered}
\leq \sum_{i=1}^{r} \sum_{j=1}^{r} h_{i} h_{j}^{k}\left[\bar{C}_{i j} \bar{x}(t)+\bar{D}_{i j} w(t)\right]^{T} \\
\times\left[\bar{C}_{i j} \bar{x}(t)+\bar{D}_{i j} w(t)\right] .
\end{gathered}
$$

It is straight forward to obtain the following results:

$$
\begin{gathered}
\dot{V}(t)+\frac{1}{\varepsilon} z^{T}(t) z(t)+\delta w^{T}(t) w(t)-2 z^{T}(t) w(t) \\
\leq \sum_{i=1}^{r} \sum_{j=1}^{r} h_{i} h_{j}^{k} \eta^{T}(t) \\
\times\left[\Phi_{11 i j}+\frac{1}{\varepsilon} \Phi_{12} i j^{T} \Phi_{12 i j}+\Phi_{13 i j}^{T} L_{i j}^{-1} \Phi_{13 i j}\right. \\
+\frac{h-d(t)}{h} \\
\times\left(\Theta_{1 i j}+\frac{1}{\varepsilon} \Phi_{14 i j}^{T} \Phi_{14 i j}-\Phi_{15 i j}^{T} \Phi_{55}^{-1} \Phi_{15 i j}\right) \\
\left.+\frac{d(t)}{h} \Theta_{2 i j}\right] \eta(t) \\
=\sum_{i=1}^{r} \sum_{j=1}^{r} h_{i} h_{j}^{k} \eta^{T}(t) \\
\times\left[\frac{h-d(t)}{h}\right. \\
\times\left(\Phi_{11 i j}+\Theta_{1 i j}+\frac{1}{\varepsilon} \Phi_{12 i j}^{T} \Phi_{12 i j}\right. \\
+\Phi_{13 i j}^{T} L_{i j}^{-1} \Phi_{13 i j}+\frac{1}{\varepsilon} \Phi_{14 i j}^{T} \Phi_{14 i j} \\
\left.-\Phi_{15 i j}^{T} \Phi_{55}^{-1} \Phi_{15 i j}\right) \\
\left.+\frac{d(t)}{h} \times\left(\Phi_{11 i j}+\Theta_{2 i j}+\frac{1}{\varepsilon} \Phi_{12 i j}^{T} \Phi_{i j}^{-1} \Phi_{13 i j}\right)\right] \eta(t) \\
\\
\times
\end{gathered}
$$

where

$$
\eta^{T}(t)=\left[\begin{array}{lllll}
x^{T}(t) & x^{T}(t-d(t)) & x^{T}(t-h) & w^{T}(t)
\end{array}\right] .
$$

By using Schur complement to LMI conditions (11)-(13) in Theorem 2, the following inequality holds:

$$
\dot{V}(t)+\frac{1}{\varepsilon} z^{T}(t) z(t)+\delta w^{T}(t) w(t)-2 z^{T}(t) z(t) \leq 0
$$

Integrating both sides of the inequality yields

$$
\begin{aligned}
& 2 \int_{0}^{t} z^{T}(s) w(s) d s \\
& \quad \geq V(t)-V(0)+\frac{1}{\varepsilon} \int_{0}^{t} z^{T}(s) z(s) d s+\delta \int_{0}^{t} w^{T}(s) w(s) d s \\
& \quad \geq \rho+\frac{1}{\varepsilon} \int_{0}^{t} z^{T}(s) z(s) d s+\delta \int_{0}^{t} w^{T}(s) w(s) d s,
\end{aligned}
$$

where $\rho=-V(0)$. From Definition 1, it can be seen that system (8) is very-strictly passive. The proof is finished.

In the following part of this section, the control gain matrices $A_{c i}, A_{c d i}, B_{c i}$, and $C_{c i}$ in (8) will be solved. Based on the LMI conditions in Theorem 2, the existence condition of controller (6) for the closed-loop system in (8) is given in the following theorem.

Theorem 3. Consider the closed-loop system (8), for given scalar $h>0, v_{R_{i j}}>0, v_{I}>0, v_{Z_{1}}>0$, and $v_{Z_{2}}>0$; the closed-loop system in (8) is very-strictly passive, if there exist scalars $\varepsilon>0, \delta>0$, matrices $\bar{P}=\bar{P}^{T}>0, \check{R}_{i j}=\check{R}_{i j}^{T}>0$, $\check{Q}_{i j}=\check{Q}_{i j}^{T}>0, \check{Z}_{1}=\check{Z}_{1}^{T}>0, \check{Z}_{2}=\check{Z}_{2}^{T}>0, \bar{I}=\bar{I}^{T}>0$, $\check{N}_{1}=\check{N}_{1}^{T}>0, \mathbb{R}=\mathbb{R}^{T}>0, \mathbb{S}=\mathbb{S}^{T}>0$, and $\mathbb{A}_{i}, \mathbb{A}_{d i}, \mathbb{B}_{i}$, $\mathbb{C}_{i}$ with appropriate dimensions, such that the following LMIs hold for $i, j=1,2, \ldots, r$ :

$$
\begin{aligned}
& {\left[\begin{array}{ccccc}
\Psi_{11 i j}+\Gamma_{1 i j} & \Psi_{12 i j} & \Psi_{13 i j} & \Psi_{14 i j} & \Psi_{15 i j} \\
\star & \Psi_{22 i j} & 0 & \Psi_{24 i j} & 0 \\
\star & \star & \Psi_{33 i j} & \Psi_{34 i j} & 0 \\
\star & \star & \star & \Psi_{44 i j} & 0 \\
\star & \star & \star & \star & \Psi_{44 i j}
\end{array}\right]<0,} \\
& {\left[\begin{array}{cccc}
\Psi_{11 i j}+\Gamma_{2 i j} & \Psi_{12 i j}^{T} & \Psi_{14 i j} & \Psi_{15 i j} \\
\star & \Psi_{22 i j} & \Psi_{24 i j} & 0 \\
\star & \star & \Psi_{44 i j} & 0 \\
\star & \star & \star & \Psi_{44 i j}
\end{array}\right]<0,} \\
& \check{Q}_{i j}<\check{L}_{i j} \text {, }
\end{aligned}
$$

where

$$
\begin{gathered}
\Psi_{11 i j}=\left[\begin{array}{cccc}
\Upsilon_{11 i j} & \Upsilon_{12 i j} & 0 & \Upsilon_{14 i j} \\
\star & \Upsilon_{22 i j} & \Upsilon_{23 i j} & 0 \\
\star & \star & \Upsilon_{33 i j} & 0 \\
\star & \star & \star & \Upsilon_{44 i j}
\end{array}\right], \\
\Psi_{12 i j}=\left[\begin{array}{cccc}
\lambda_{4 i j}^{T}+\lambda_{4 j i}^{T} & 0 & 0 & \bar{D}_{w i j}^{T}+\bar{D}_{w j i}^{T} \\
h \lambda_{1 i j} & h \lambda_{2 i j} & 0 & \lambda_{3 i j}+\lambda_{3 j i}
\end{array}\right]^{T}, \\
\Gamma_{1 i j}=\left[\begin{array}{cccc}
-\bar{Q}_{i j} & \bar{Q}_{i j} & 0 & 0 \\
\bar{Q}_{i j} & -\bar{Q}_{i j} & 0 & 0 \\
0 & 0 & 0 & 0 \\
0 & 0 & 0 & 0
\end{array}\right],
\end{gathered}
$$




$$
\begin{aligned}
& \Gamma_{2 i j}=\left[\begin{array}{cccc}
0 & 0 & 0 & 0 \\
0 & -\bar{Q}_{i j} & \bar{Q}_{i j} & 0 \\
0 & \bar{Q}_{i j} & -\bar{Q}_{i j} & 0 \\
0 & 0 & 0 & 0
\end{array}\right] \\
& \Psi_{13 i j}=\left[\begin{array}{cccc}
2 h \nu_{Z_{2}} \Delta & 2 h \nu_{Z_{2}} \Delta & 0 & 0 \\
h \lambda_{1 i j} & h \lambda_{2 i j} & 0 & h \lambda_{3 i j}+h \lambda_{3 j i} \\
h \lambda_{1 i j} & h \lambda_{2 i j} & 0 & h \lambda_{3 i j}+h \lambda_{3 j i}
\end{array}\right]^{T}, \\
& \Psi_{14 i j}=\left[\begin{array}{llll}
\mu_{1 i j} & 0 & 0 & 0
\end{array}\right]^{T} \text {, } \\
& \Psi_{15 i j}=\left[\begin{array}{llll}
\mu_{2 i j} & \mu_{3 i j} & 0 & 0
\end{array}\right]^{T} \text {, } \\
& \Psi_{44 i j}=\left[\begin{array}{cc}
-I & 0 \\
0 & -I
\end{array}\right], \quad \Psi_{24 i j}=\left[\begin{array}{ll}
0 & h \mu_{1 i j}
\end{array}\right]^{T}, \\
& \Psi_{22 i j}=\operatorname{diag}\left\{-2 \varepsilon I, \Pi_{1 i j}\right\} \text {, } \\
& \Psi_{33 i j}=\operatorname{diag}\left\{2 h \Pi_{2 i j}, 2 h \Pi_{2 i j}, 2 h \Pi_{3 i j}\right\}, \\
& \Psi_{34 i j}=\left[\begin{array}{lll}
0 & h \mu_{1 i j} & h \mu_{1 i j}
\end{array}\right]^{T}, \\
& \Upsilon_{12 i j}=\lambda_{2 i j}+\check{Q}_{i j}+\check{Q}_{j i}+\frac{2}{h} \check{Z}_{1}+2 v_{Z_{2}} \Delta, \\
& \Upsilon_{11 i j}=\left[\lambda_{1 i j}\right]_{s}-\check{Q}_{i j}-\check{Q}_{j i}-\frac{2}{h} \check{Z}_{1}-2 v_{Z_{2}} \Delta-2 \check{N}_{1} \text {, } \\
& \Upsilon_{14 i j}=\lambda_{3 i j}-\lambda_{4 i j}+\lambda_{3 j i}-\lambda_{4 j i} \text {, } \\
& \Upsilon_{23 i j}=-\check{Q}_{i j}-\check{Q}_{j i} \text {, } \\
& \Upsilon_{22 i j}=-\check{Q}_{i j}-\check{Q}_{j i}-\frac{2}{h} \check{Z}_{1}-2 v_{Z_{2}} \Delta \text {, } \\
& \Upsilon_{33 i j}=-\check{Q}_{i j}-\check{Q}_{j i}-2 \check{N}_{1} \text {, } \\
& \Upsilon_{33 i j}=2 \delta I-\bar{D}_{w i j}-\bar{D}_{w i j}^{T}-\bar{D}_{w j i}-\bar{D}_{w j i}^{T} \text {, } \\
& \Pi_{2 i j}=v_{I}^{2} \bar{I}-2 h v_{I} \Delta, \\
& \Pi_{1 i j}=v_{L_{i j}}^{2} \bar{L}_{i j}+v_{L_{j i}}^{2} \bar{L}_{j i}-2 v_{L_{i j}} \Delta-2 v_{L_{j i}} \Delta, \\
& \Pi_{3 i j}=v_{Z_{1}}^{2} \bar{Z}_{1}-4 h v_{Z_{1}} \Delta, \\
& \lambda_{1 i j}=\left[\begin{array}{cc}
\mathbb{R} A_{i}+\mathbb{R} A_{j} & \mathbb{A}_{i}+\mathbb{A}_{j} \\
A_{i}+A_{j} & A_{i} \mathbb{S}+B_{i} \mathbb{C}_{j}+A_{j} \mathbb{S}+B_{j} \mathbb{C}_{i}
\end{array}\right], \\
& \mu_{1 i j}^{T}=\left[\begin{array}{cc}
\mathbb{R}\left(B_{i}-B_{j}\right) & \mathbb{B}_{j}-\mathbb{B}_{i} \\
0 & 0
\end{array}\right], \\
& \lambda_{2 i j}=\left[\begin{array}{cc}
\mathbb{B}_{j} C_{y i}+\mathbb{B}_{i} C_{y j} & \mathbb{A}_{d i}+\mathbb{A}_{d j} \\
0 & 0
\end{array}\right] \text {, } \\
& \lambda_{3 i j}=\left[\begin{array}{c}
\mathbb{R} B_{w i} \\
B_{w i}
\end{array}\right], \quad \lambda_{4 i j}=\left[\begin{array}{c}
C_{i}^{T} \\
\mathbb{S} C_{i}^{T}+\mathbb{C}_{j} D_{i}^{T}
\end{array}\right], \\
& \mu_{2 i j}=\left[\begin{array}{cc}
0 & 0 \\
\mathbb{C}_{j}^{T}-\mathbb{C}_{i}^{T} & 0
\end{array}\right]^{T}
\end{aligned}
$$

$$
\begin{gathered}
\mu_{3 i j}=\left[\begin{array}{cc}
0 & 0 \\
0 & \mathbb{S}\left(C_{y i}-C_{y j}\right)^{T}
\end{array}\right]^{T}, \quad \Delta=\left[\begin{array}{cc}
\mathbb{R} & I \\
I & \mathbb{S}
\end{array}\right], \\
\check{N}_{1}=\left[\begin{array}{cc}
N_{11} & N_{12} \\
\star & N_{13}
\end{array}\right], \quad \check{Z}_{1}=\left[\begin{array}{cc}
Z_{11} & Z_{12} \\
\star & Z_{13}
\end{array}\right], \\
\check{Z}_{2}=\left[\begin{array}{cc}
Z_{21} & Z_{22} \\
\star & Z_{23}
\end{array}\right], \\
\check{R}_{i j}=\left[\begin{array}{cc}
R_{1 i j} & R_{2 i j} \\
\star & R_{3 i j}
\end{array}\right], \quad \check{Q}_{i j}=\left[\begin{array}{cc}
Q_{1 i j} & Q_{2 i j} \\
\star & Q_{3 i j}
\end{array}\right], \\
\bar{I}=\left[\begin{array}{cc}
I_{1} & I_{2} \\
\star & I_{3}
\end{array}\right] .
\end{gathered}
$$

Thus, the dynamic output-feedback control gain matrices can be obtained as shown below:

$$
\begin{gathered}
A_{c i}=\mathbb{M}^{-1}\left(\mathbb{A}_{i}-\mathbb{R} B_{i} \mathbb{C}_{i}-\mathbb{R} A_{i} \mathbb{S}\right) \mathbb{N}^{-T}, \\
A_{c d i}=\mathbb{M}^{-1}\left(\mathbb{A}_{d i}-\mathbb{B} C_{y i} \mathbb{S}\right) \mathbb{N}^{-T} \\
B_{c i}=\mathbb{M}^{-1} \mathbb{B}_{i}, \\
C_{c i}=\mathbb{C}_{i} \mathbb{N}^{-T},
\end{gathered}
$$

where $\mathbb{M}$ and $\mathbb{N}$ are nonsingular matrices satisfying

$$
\mathbb{M N}^{T}=I-\mathbb{R} \mathbb{S} \text {. }
$$

Proof. By the Schur complement, it can be seen that (26) is equivalent to

$$
\left[\begin{array}{ccc}
\Psi_{11 i j}+\Gamma_{1 i j} & \Psi_{12 i j} & \Psi_{13 i j} \\
\star & \Psi_{22 i j} & 0 \\
\star & \star & \Psi_{33 i j}
\end{array}\right]+\alpha \alpha^{T}+\beta \beta^{T}+\varsigma \varsigma^{T}+\omega \omega^{T}<0
$$

where

$$
\begin{aligned}
& \alpha^{T}=\left[\left(B_{i}-B_{j}\right)^{T} \mathbb{R}^{T} 0_{1 \times 7} h\left(B_{i}-B_{j}\right)^{T} \mathbb{R}^{T} 0_{1 \times 3}\right. \\
& \left.h\left(B_{i}-B_{j}\right)^{T} \mathbb{R}^{T} \quad 0 \quad h\left(B_{i}-B_{j}\right)^{T} \mathbb{R}^{T} \quad 0\right], \\
& \beta^{T}=\left[\begin{array}{lll}
\left(\mathbb{B}_{j}-\mathbb{B}_{i}\right)^{T} & 0_{1 \times 7} & h\left(\mathbb{B}_{j}-\mathbb{B}_{i}\right)^{T} \quad 0_{1 \times 3}
\end{array}\right. \\
& \left.h\left(\mathbb{B}_{j}-\mathbb{B}_{i}\right)^{T} \quad 0 \quad h\left(\mathbb{B}_{j}-\mathbb{B}_{i}\right)^{T} \quad 0\right], \\
& \varsigma^{T}=\left[\begin{array}{lll}
0 & \left(\mathbb{C}_{j}-\mathbb{C}_{i}\right) & 0_{1 \times 14}
\end{array}\right], \\
& \omega^{T}=\left[\begin{array}{lll}
0_{1 \times 3} & \left(C_{y i}-C_{y j}\right) \mathbb{S}^{T} & 0_{1 \times 12}
\end{array}\right] .
\end{aligned}
$$

It is clear to see that

$$
\alpha \alpha^{T}+\varsigma \varsigma^{T}+\beta \beta^{T}+\omega \omega^{T} \geq \alpha \varsigma^{T}+\varsigma \alpha^{T}+\beta \omega^{T}+\omega \beta^{T},
$$

which means

$$
\left[\begin{array}{ccc}
\Psi_{11 i j}+\Gamma_{1 i j} & \Psi_{12 i j} & \Psi_{13 i j} \\
\star & \check{\Xi}_{22 i j} & 0 \\
\star & \star & \check{\Xi}_{33 i j}
\end{array}\right]+\alpha \varsigma^{T}+\varsigma \alpha^{T}+\beta \omega^{T}+\omega \beta^{T}<0 .
$$


By the output-feedback controller, the matrix $\bar{P}$ is partitioned and inverted as

$$
\bar{P}=\left[\begin{array}{cc}
\mathbb{S} & \mathbb{N} \\
\mathbb{N}^{T} & \mathbb{Y}
\end{array}\right], \quad \bar{P}^{-1}=\left[\begin{array}{cc}
\mathbb{R} & \mathbb{M} \\
\mathbb{M}^{T} & \mathbb{T}
\end{array}\right]
$$

It can be seen that (32) holds via $\overline{P P}^{-1}=I$. According to Schur complement formula, it implies that $\mathbb{R}-\mathbb{S}^{-1}>0$; therefore $\mathbb{R S}-I$ is nonsingular. This shows that there exist nonlinear matrices $\mathbb{N}$ and $\mathbb{M}$ such that (32) is satisfied. Set

$$
\Omega_{1}=\left[\begin{array}{cc}
\mathbb{R} & I \\
\mathbb{M}^{T} & 0
\end{array}\right], \quad \Omega_{2}=\left[\begin{array}{cc}
I & \mathbb{S} \\
0 & \mathbb{N}^{T}
\end{array}\right] .
$$

Then, we obtain from (38) that

$$
\bar{P} \Omega_{1}=\Omega_{2}
$$

It follows that

$$
\Omega_{1}^{T} \bar{P} \Omega_{1}=\Omega_{1}^{T} \Omega_{2}=\left[\begin{array}{cc}
\mathbb{R} & I \\
I & \mathbb{S}
\end{array}\right],
$$

which shows that the matrices $\Omega_{1}$ and $\Omega_{2}$ in (39) are positive definite. It can be found that the matrix $\bar{P}$ can be expressed as $\bar{P}=\Omega_{2} \Omega_{1}^{-1}$, and it is known that $\bar{P}>0$. The following equations can be obtained:

$$
\begin{aligned}
& \mathbb{R} A_{i} \mathbb{S}+\mathbb{R} B_{i} \mathbb{C}_{i}+\mathbb{M} A_{c i} \mathbb{N}^{T}+\mathbb{R} A_{j} \mathbb{S}+\mathbb{R} B_{j} \mathbb{C}_{j} \\
&+ \mathbb{M} A_{c j} \mathbb{N}^{T}+\mathbb{R}\left(B_{i}-B_{j}\right)\left(\mathbb{C}_{j}-\mathbb{C}_{i}\right) \\
&= \mathbb{R} A_{i} \mathbb{S}+\mathbb{R} B_{i} \mathbb{C}_{j}+\mathbb{M} A_{c j} \mathbb{N}^{T}+\mathbb{R} A_{j} \mathbb{S}+\mathbb{R} B_{j} \mathbb{C}_{i} \\
&+\mathbb{M} A_{c i} \mathbb{N}^{T}, \\
& \mathbb{B}_{i} C_{y i} \mathbb{S}+\mathbb{M} A_{c d i} \mathbb{N}^{T}+\mathbb{B}_{j} C_{y j} \mathbb{S}+\mathbb{M} A_{c d j} \mathbb{N}^{T} \\
&+\left(\mathbb{B}_{j}-\mathbb{B}_{i}\right)\left(C_{y i}-C_{y j}\right) \mathbb{S} \\
&=\mathbb{B}_{i} C_{y j} \mathbb{S}+\mathbb{M} A_{c d j} \mathbb{N}^{T}+\mathbb{B}_{j} C_{y i} \mathbb{S}+\mathbb{M} A_{c d i} \mathbb{N}^{T} .
\end{aligned}
$$

Because of the nonsingular matrices $\mathbb{M}$ and $\mathbb{N}$, the control gain matrices $A_{c i}, A_{c d i}, B_{c i}$, and $C_{c i}$ can be solved from (31). Then, by performing a congruent transformation to (36) by $\operatorname{diag}\left\{\Omega_{1}^{-1}, \Omega_{1}^{-1}, \Omega_{1}^{-1}, I, I, \Omega_{1}^{-1}, \Omega_{1}^{-1}, \Omega_{1}^{-1}, \Omega_{1}^{-1}\right\}$, the following inequality holds:

$$
\left[\begin{array}{ccc}
\Xi_{11 i j}+\Xi_{11 j i}+\bar{\Theta}_{1 i j}+\bar{\Theta}_{1 j i} & \Xi_{12 i j}+\Xi_{12 j i} & \Xi_{13 i j}+\Xi_{13 j i} \\
\star & \Xi_{22 i j}+\Xi_{22 j i} & 0 \\
\star & \star & \Xi_{33 i j}+\Xi_{33 j o}
\end{array}\right]<0,
$$

where

$$
\begin{aligned}
& \Xi_{11 i j}=\left[\begin{array}{cccc}
\bar{\Phi}_{11 i j} & \bar{\Phi}_{12 i j} & 0 & \bar{\Phi}_{14 i j} \\
\star & \bar{\Phi}_{22 i j} & \bar{Q}_{i j} & 0 \\
\star & \star & \bar{\Phi}_{33 i j} & 0 \\
\star & \star & \star & \bar{\Phi}_{44 i j}
\end{array}\right] \text {, } \\
& \bar{\Phi}_{11 i j}=\left[\bar{A}_{i j} \bar{P}\right]_{s}-\bar{Q}_{i j}-\frac{1}{h} \bar{Z}_{1}-v_{Z_{2}} \bar{P}+\bar{N}_{1}, \\
& \bar{\Phi}_{12 i j}=\bar{B}_{i j} \bar{P}+\bar{Q}_{i j}+\frac{1}{h} \bar{Z}_{1}+v_{Z_{2}} \bar{P}, \\
& \bar{\Phi}_{14 i j}=\bar{B}_{w i j}-\bar{P} \bar{C}_{i j}^{T}, \quad \bar{\Phi}_{22 i j}=-2 \bar{Q}_{i j}-\frac{1}{h} \bar{Z}_{1}-v_{Z_{2}} \bar{P}, \\
& \bar{\Phi}_{33 i j}=-\bar{Q}_{i j}-\bar{N}_{1}, \quad \bar{\Phi}_{44 i j}=\delta I-\bar{D}_{w i j}-\bar{D}_{w i j}^{T}, \\
& \Xi_{22 i j}=\operatorname{diag}\left\{-\varepsilon I, v_{R i j}^{2} \bar{R}_{i j}-2 v_{R i j} \bar{P}\right\}, \\
& \Xi_{33 i j}=\operatorname{diag}\left\{v_{I}^{2} \bar{I}-2 v_{I} \bar{P}, v_{I}^{2} \bar{I}-2 v_{I} \bar{P}, h\left(v_{Z_{1}}^{2} \bar{Z}_{1}-2 v_{Z_{1}} \bar{P}\right)\right\}, \\
& \Xi_{12 i j}=\left[\begin{array}{cccc}
\bar{C}_{i j} \bar{P} & 0 & 0 & \bar{D}_{w i j} \\
h \bar{A}_{i j} \bar{P} & h \bar{B}_{i j} \bar{P} & 0 & h \bar{B}_{w i j}
\end{array}\right]^{T}, \\
& \Xi_{13 i j}=\left[\begin{array}{cccc}
h v_{Z 2} \bar{P} & h v_{Z 2} \bar{P} & 0 & 0 \\
h \bar{A}_{i j} \bar{P} & h \bar{B}_{i j} \bar{P} & 0 & h \bar{B}_{w i j} \\
h \bar{A}_{i j} \bar{P} & h \bar{B}_{i j} \bar{P} & 0 & h \bar{B}_{w i j}
\end{array}\right]^{T} .
\end{aligned}
$$

It can be seen from $v_{Z_{1}}>0$ that

$$
\left(v_{Z_{1}} \bar{Z}_{1}-\bar{P}\right) \bar{Z}_{1}^{-1}\left(v_{Z_{1}} \bar{Z}_{1}-\bar{P}\right) \geq 0
$$

which means

$$
-\bar{P} \bar{Z}_{1}^{-1} \bar{P} \leq v_{Z_{1}}^{2} \bar{Z}_{1}-2 v_{Z_{1}} \bar{P} .
$$

Similarly

$$
\begin{gathered}
-\bar{P} \bar{R}_{i j}^{-1} \bar{P} \leq v_{R_{i j}}^{2} \bar{R}_{i j}-2 v_{R_{i j}} \bar{P}, \\
-\bar{P} \bar{I}^{-1} \bar{P} \leq v_{I}^{2} \bar{I}-2 v_{I} \bar{P} .
\end{gathered}
$$

Then, we know that

$$
\left[\begin{array}{ccc}
\Xi_{11 i j}+\Xi_{11 j i}+\bar{\Theta}_{1 i j}+\bar{\Theta}_{1 j i} & \Xi_{12 i j}+\Xi_{12 j i} & \Xi_{13 i j}+\Xi_{13 j i} \\
\star & \bar{\Xi}_{22 i j}+\bar{\Xi}_{22 j i} & 0 \\
\star & \star & \bar{\Xi}_{33 i j}+\bar{\Xi}_{33 j i}
\end{array}\right]
$$


where

$$
\begin{gathered}
\bar{\Xi}_{22 i j}=\operatorname{diag}\left\{-\varepsilon I,-\bar{P} \bar{R}_{i j}^{-1} \bar{P}\right\}, \\
\bar{\Xi}_{33 i j}=\operatorname{diag}\left\{-\bar{P} \bar{I}^{-1} \bar{P},-\bar{P} \bar{I}^{-1} \bar{P},-h \bar{P} \bar{Z}_{1}^{-1} \bar{P}\right\} .
\end{gathered}
$$

For (47), through the congruence transformations by $\operatorname{diag}\left\{P, P, P, I, I, R_{i j}, I, I, Z_{1}\right\}$ with the change of matrix variables defined by $P=\bar{P}^{-1}, R_{i j}=\bar{P}^{-1} \bar{R}_{i j} \bar{P}^{-1}, Z_{1}=\bar{P}^{-1} \bar{Z}_{1} \bar{P}^{-1}$ and $I=\bar{P}^{-1} \bar{I} \bar{P}^{-1}$, one has
Similarly,

$$
\left[\begin{array}{ccc}
\Phi_{11 i j}+\Phi_{11 j i}+\Theta_{2 i j}+\Theta_{2 j i} & \Phi_{12 i j}+\Phi_{12 j i} & \Phi_{13 i j}+\Phi_{13 j i} \\
\star & -2 \varepsilon I & 0 \\
\star & \star & -L_{i j}-L_{j i}
\end{array}\right]
$$$$
<0 \text {. }
$$

It is shown that

$$
\begin{gathered}
{\left[\begin{array}{ccccc}
\Phi_{11 i j}+\Theta_{1 i j} & \Phi_{12 i j} & \Phi_{13 i j} & \Phi_{14 i j} & \Phi_{15 i j} \\
\star & -\varepsilon I & 0 & 0 & 0 \\
\star & \star & -L_{i j} & 0 & 0 \\
\star & \star & \star & -\varepsilon I & 0 \\
\star & \star & \star & \star & \Phi_{55 i j}
\end{array}\right]<0,} \\
{\left[\begin{array}{cccc}
\Phi_{11 i j}+\Theta_{2 i j} & \Phi_{12 i j} & \Phi_{13 i j} \\
\star & & -\varepsilon I & 0 \\
\star & & \star & -L_{i j}
\end{array}\right]<0 .}
\end{gathered}
$$

Therefore, all the conditions in Theorem 2 are satisfied. The proof is completed.

\section{Numerical Example}

In this section, a numerical example is given to show the effectiveness of the proposed results. We consider the follow fuzzy system.

Plant Rule 1. IF $x_{1}(t)$ is $h_{1}(\theta(t))$, THEN

$$
\begin{gathered}
\dot{x}(t)=A_{1} x(t)+B_{1} u(t)+B w_{1} w(t), \\
z(t)=C_{1} x(t)+D_{1} u(t)+D w_{1} w(t), \\
y(t)=C_{y 1} x(t) .
\end{gathered}
$$

Plant Rule 2. IF $x_{1}(t)$ is $h_{2}(\theta(t))$, THEN

$$
\begin{gathered}
\dot{x}(t)=A_{2} x(t)+B_{2} u(t)+B w_{2} w(t), \\
z(t)=C_{2} x(t)+D_{2} u(t)+D w_{2} w(t), \\
y(t)=C_{y 2} x(t),
\end{gathered}
$$

where

$$
\begin{gathered}
A_{1}=\left[\begin{array}{cc}
0 & 0.7 \\
-0.314 & 1
\end{array}\right], \quad A_{2}=\left[\begin{array}{cc}
0 & 0.3 \\
0.1 & -0.256
\end{array}\right], \\
B_{1}=\left[\begin{array}{c}
0 \\
0.1
\end{array}\right], \quad B_{2}=\left[\begin{array}{c}
0.1 \\
0
\end{array}\right], \\
B_{w 1}=\left[\begin{array}{ll}
-1 & 0
\end{array}\right]^{T}, \quad B_{w 2}=\left[\begin{array}{ll}
0 & -1
\end{array}\right]^{T}, \\
C_{1}=\left[\begin{array}{ll}
1 & 0
\end{array}\right], \quad C_{2}=\left[\begin{array}{ll}
-1 & 0
\end{array}\right], \quad D_{1}=0.01, \\
C_{y 1}=\left[\begin{array}{ll}
1 & 0.5
\end{array}\right], \quad C_{y 2}=\left[\begin{array}{ll}
-0.5 & 1
\end{array}\right], \\
D_{2}=0.02, \quad D_{w 1}=0.1, \quad D_{w 2}=0.2 .
\end{gathered}
$$

For $h=0.05, h_{1}(\theta(t))=e^{\left(1-x_{2}\right)^{2}} /\left(e^{\left(1-x_{2}\right)^{2}}+e^{\left(-x_{2}\right)^{2}}\right)$, and $h_{2}(\theta(t))=1-h_{1}(\theta(t))$, it is found that $\delta=13, \varepsilon=12$, and the system with output-feedback sampled-delay controller meets the passive performance. Then the dynamic output-feedback control gain matrices can be obtained as shown below:

$$
\begin{aligned}
A_{c 1} & =\left[\begin{array}{ll}
3.6932 & 7.1253 \\
3.0405 & 9.5623
\end{array}\right], \\
A_{c 2} & =\left[\begin{array}{ll}
4.9324 & 25.7304 \\
3.9106 & 21.1046
\end{array}\right], \\
B_{c 1} & =10^{-4} \times\left[\begin{array}{c}
-0.5860 \\
0.0185
\end{array}\right], \\
B_{c 2} & =10^{-4} \times\left[\begin{array}{cc}
-0.2391 \\
-0.3317
\end{array}\right], \\
A_{c d 1} & =\left[\begin{array}{ll}
-0.0098 & 0.2607 \\
0.0008 & -0.0277
\end{array}\right], \\
A_{c d 2} & =\left[\begin{array}{ll}
0.0003 & -0.0105 \\
0.0063 & -0.1557
\end{array}\right], \\
C_{c 1} & =\left[\begin{array}{ll}
-3.5198 & -18.1033
\end{array}\right], \\
C_{c 2} & =\left[\begin{array}{ll}
-3.9239 & -23.6327
\end{array}\right] .
\end{aligned}
$$




\section{Conclusions}

In this paper, the problem of dynamic output-feedback control has been investigated for a class of nonlinear systems via T-S fuzzy control approach. By using Lyapunov stability theory, a sufficient condition for very-strict passive performance analysis for fuzzy systems with nonuniform uncertain sampling has been derived. Based on the condition, a novel fuzzy dynamic output-feedback controller has been designed such that the closed-loop system is very-strictly passive. The existence condition of the controller has been solved by convex optimization approach. Finally, a numerical example has been provided to demonstrate the effectiveness of the proposed method. In future work, the fault-tolerant control problems $[40,41]$ will be investigated for fuzzy systems via dynamic output-feedback control design method.

\section{Conflict of Interests}

The authors declare that there is no conflict of interests regarding the publication of this paper.

\section{Acknowledgments}

This work was partially supported by the National Natural Science Foundation of China (61203002, 61304003, and 61304054), the Program for New Century Excellent Talents in University, the Program for Liaoning Innovative Research Team in University (LT2013023), and the Program for Liaoning Excellent Talents in University (LR2013053).

\section{References}

[1] S. Tong and H.-X. Li, "Fuzzy adaptive sliding-mode control for MIMO nonlinear systems," IEEE Transactions on Fuzzy Systems, vol. 11, no. 3, pp. 354-360, 2003.

[2] S.-C. Tong, X.-L. He, and H.-G. Zhang, "A combined backstepping and small-gain approach to robust adaptive fuzzy output feedback control," IEEE Transactions on Fuzzy Systems, vol. 17, no. 5, pp. 1059-1069, 2009.

[3] S. Tong and Y. Li, "Observer-based fuzzy adaptive control for strict-feedback nonlinear systems," Fuzzy Sets and Systems, vol. 160, no. 12, pp. 1749-1764, 2009.

[4] Y. Li, S. Tong, Y. Liu, and T. Li, "Adaptive fuzzy robust output feedback control of nonlinear systems with unknown dead zones based on small-gain approach," IEEE Transactions on Fuzzy Systems, 2013.

[5] Q. Zhou, P. Shi, J. Lu, and S. Xu, "Adaptive output-feedback fuzzy tracking control for a class of nonlinear systems," IEEE Transactions on Fuzzy Systems, vol. 19, no. 5, pp. 972-982, 2011.

[6] T. Takagi and M. Sugeno, "Fuzzy identification of systems and its applications to modeling and control," IEEE Transactions on Systems, Man and Cybernetics, vol. 15, no. 1, pp. 116-132, 1985.

[7] G. Feng, "A survey on analysis and design of model-based fuzzy control systems," IEEE Transactions on Fuzzy Systems, vol. 14, no. 5, pp. 676-697, 2006.

[8] M. Liu, X. Cao, and P. Shi, "Fault estimation and tolerant control for fuzzy stochastic systems," IEEE Transactions on Fuzzy Systems, vol. 21, no. 2, pp. 221-229, 2013.
[9] J. Qiu, G. Feng, and H. Gao, "Asynchronous output-feedback control of networked nonlinear systems with multiple packet dropouts: T-S fuzzy affine model-based approach," IEEE Transactions on Fuzzy Systems, vol. 19, no. 6, pp. 1014-1030, 2011.

[10] K. Tanaka and H. O. Wang, Fuzzy Control Systems Design and Analysis. A Linear Matrix Inequality Approach, John Wiley \& Sons, New York, NY, USA, 2001.

[11] X. Su, P. Shi, L. Wu, and S. K. Nguang, "Induced $\ell_{2}$ filtering of fuzzy stochastic systems with time-varying delays," IEEE Transactions on Cybernetics, vol. 43, no. 4, pp. 1251-1264, 2013.

[12] M. Liu, X. Cao, and P. Shi, "Fuzzy-model-based fault tolerant design for nonlinear stochastic systems against simultaneous sensor and actuator faults," IEEE Transactions on Fuzzy Systems, 2013.

[13] B. Chen, X. Liu, and S. Tong, "Delay-dependent stability analysis and control synthesis of fuzzy dynamic systems with time delay," Fuzzy Sets and Systems, vol. 157, no. 16, pp. 22242240, 2006.

[14] L. Wu, X. Su, P. Shi, and J. Qiu, "A new approach to stability analysis and stabilization of discrete-time T-S fuzzy timevarying delay systems," IEEE Transactions on Systems, Man, and Cybernetics B, vol. 41, no. 1, pp. 273-286, 2011.

[15] H. Li, B. Chen, C. Lin, and Q. Zhou, "Mean square exponential stability of stochastic fuzzy Hopfield neural networks with discrete and distributed time-varying delays," Neurocomputing, vol. 72, no. 7-9, pp. 2017-2023, 2009.

[16] B. Chen, X. Liu, C. Lin, and K. Liu, "Robust $H_{\infty}$ control of Takagi-Sugeno fuzzy systems with state and input time delays," Fuzzy Sets and Systems, vol. 160, no. 4, pp. 403-422, 2009.

[17] S. Xu and J. Lam, "Robust $H_{\infty}$ control for uncertain discretetime-delay fuzzy systems via output feedback controllers," IEEE Transactions on Fuzzy Systems, vol. 13, no. 1, pp. 82-93, 2005.

[18] C. Lin, Q.-G. Wang, T. H. Lee, and Y. He, LMI Approach to Analysis and Control of Takagi-Sugeno Fuzzy Systems with Time Delay, vol. 351 of Lecture Notes in Control and Information Sciences, Springer, Berlin, Germany, 2007.

[19] B. Zhang, J. Lam, S. Xu, and Z. Shu, "Robust stabilization of uncertain T-S fuzzy time-delay systems with exponential estimates," Fuzzy Sets and Systems, vol. 160, no. 12, pp. 1720-1737, 2009.

[20] L. Wu and W. X. Zheng, " $L_{2}-L_{\infty}$ control of nonlinear fuzzy itô stochastic delay systems via dynamic output feedback," IEEE Transactions on Systems, Man, and Cybernetics B, vol. 39, no. 5, pp. 1308-1315, 2009.

[21] H. Li, J. Yu, C. Hilton, and H. Liu, "Adaptive sliding mode control for nonlinear active suspension vehicle systems using TS fuzzy approach," IEEE Transactions on Industrial Electronics, vol. 60 , no. 8, pp. 3328-3338, 2013.

[22] L. Wu and D. W. C. Ho, "Fuzzy filter design for Itô stochastic systems with application to sensor fault detection," IEEE Transactions on Fuzzy Systems, vol. 17, no. 1, pp. 233-242, 2009.

[23] H. Li, B. Chen, Q. Zhou, and W. Qian, "Robust stability for uncertain delayed fuzzy Hopfield neural networks with Markovian jumping parameters," IEEE Transactions on Systems, Man, and Cybernetics B, vol. 39, no. 1, pp. 94-102, 2009.

[24] H. Li, H. Liu, H. Gao, and P. Shi, "Reliable fuzzy control for active suspension systems with actuator delay and fault," IEEE Transactions on Fuzzy Systems, vol. 20, no. 2, pp. 342-357, 2012.

[25] T. Chen and B. Francis, Optimal Sampled-Data Control Systems, Springer, London, UK, 1995. 
[26] H. Gao, T. Chen, and J. Lam, "A new delay system approach to network-based control," Automatica, vol. 44, no. 1, pp. 39-52, 2008.

[27] B. Bamieh, J. B. Pearson, B. A. Francis, and A. Tannenbaum, "A lifting technique for linear periodic systems with applications to sampled-data control," Systems and Control Letters, vol. 17, no. 2, pp. 79-88, 1991.

[28] T. Chen and L. Qiu, " $H_{\infty}$ design of general multirate sampleddata control systems," Automatica, vol. 30, no. 7, pp. 1139-1152, 1994.

[29] S. K. Nguang and P. Shi, "Nonlinear $\mathscr{H}_{\infty}$ filtering of sampleddata systems," Automatica, vol. 36, no. 2, pp. 303-310, 2000.

[30] E. Fridman, A. Seuret, and J.-P. Richard, "Robust sampleddata stabilization of linear systems: an input delay approach," Automatica, vol. 40, no. 8, pp. 1441-1446, 2004.

[31] E. Fridman, "A refined input delay approach to sampled-data control," Automatica, vol. 46, no. 2, pp. 421-427, 2010.

[32] H. Gao and T. Chen, "Stabilization of nonlinear systems under variable sampling: a fuzzy control approach," IEEE Transactions on Fuzzy Systems, vol. 15, no. 5, pp. 972-983, 2007.

[33] X.-L. Zhu, B. Chen, D. Yue, and Y. Wang, "An improved input delay approach to stabilization of fuzzy systems under variable sampling," IEEE Transactions on Fuzzy Systems, vol. 20, no. 2, pp. 330-341, 2012.

[34] H. K. Lam and F. H. F. Leung, "Sampled-data fuzzy controller for time-delay nonlinear systems: fuzzy-model-based LMI approach," IEEE Transactions on Systems, Man, and Cybernetics $B$, vol. 37, no. 3, pp. 617-629, 2007.

[35] H. K. Lam, "Sampled-data fuzzy-model-based control systems: stability analysis with consideration of analogue-to-digital converter and digital-to-analogue converter," IET Control Theory and Applications, vol. 4, no. 7, pp. 1131-1144, 2010.

[36] S. K. Nguang and P. Shi, "Fuzzy $\mathscr{H}_{\infty}$ output feedback control of nonlinear systems under sampled measurements," Automatica, vol. 39, no. 12, pp. 2169-2174, 2003.

[37] C. Li, H. Zhang, and X. Liao, "Passivity and passification of fuzzy systems with time delays," Computers and Mathematics with Applications, vol. 52, no. 6-7, pp. 1067-1078, 2006.

[38] J. Liang, Z. Wang, and X. Liu, "Robust passivity and passification of stochastic fuzzy time-delay systems," Information Sciences, vol. 180, no. 9, pp. 1725-1737, 2010.

[39] B. Zhang, W. X. Zheng, and S. Xu, "Passivity analysis and passive control of fuzzy systems with time-varying delays," Fuzzy Sets and Systems, vol. 174, no. 1, pp. 83-98, 2011.

[40] S. Yin, H. Luo, and S. Ding, "Real-time implementation of fault tolerant control system with performance optimization," IEEE Transactions on Industrial Electronics, 2013.

[41] S. Yin, S. Ding, A. Haghani, H. Hao, and P. Zhang, "A comparison study of basic data-driven fault diagnosis and process monitoring methods on the benchmark Tennessee Eastman process," Journal of Process Control, vol. 22, no. 9, pp. 1567-1581, 2012. 


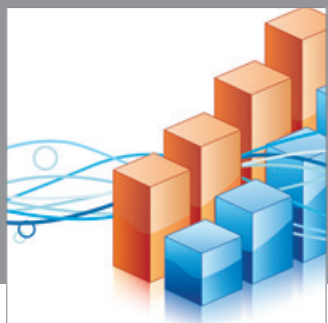

Advances in

Operations Research

mansans

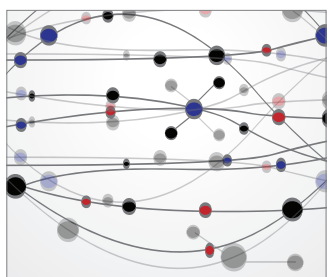

The Scientific World Journal
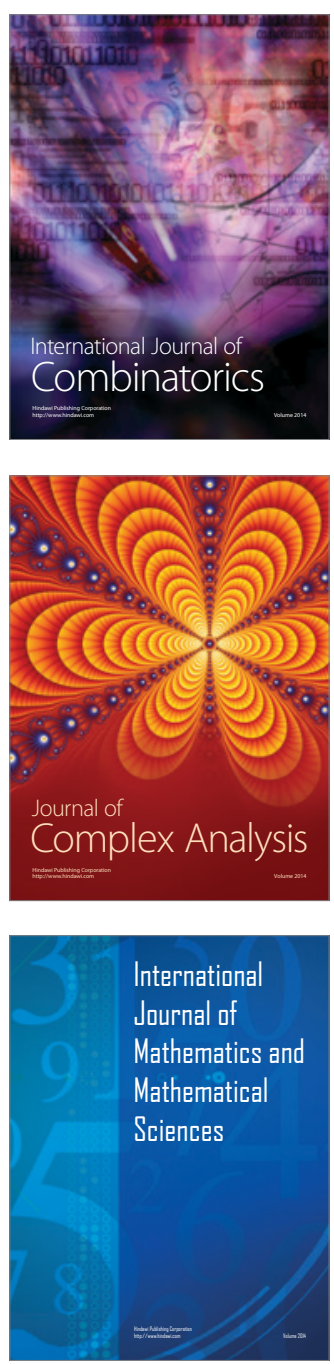


Submit your manuscripts at http://www.hindawi.com
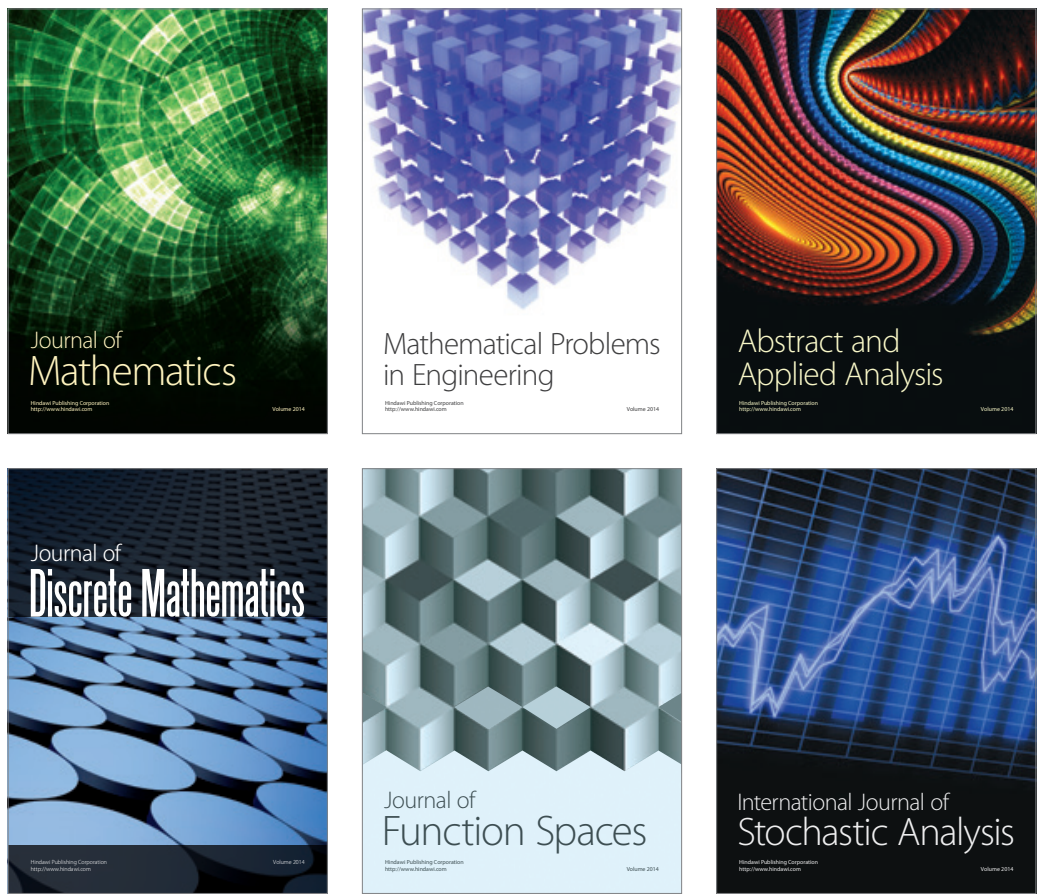

Journal of

Function Spaces

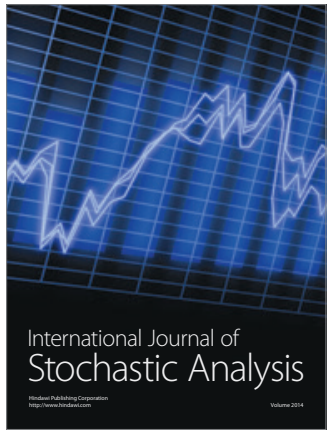

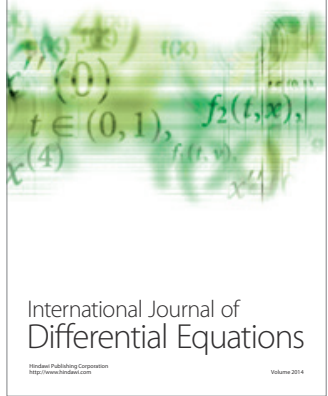
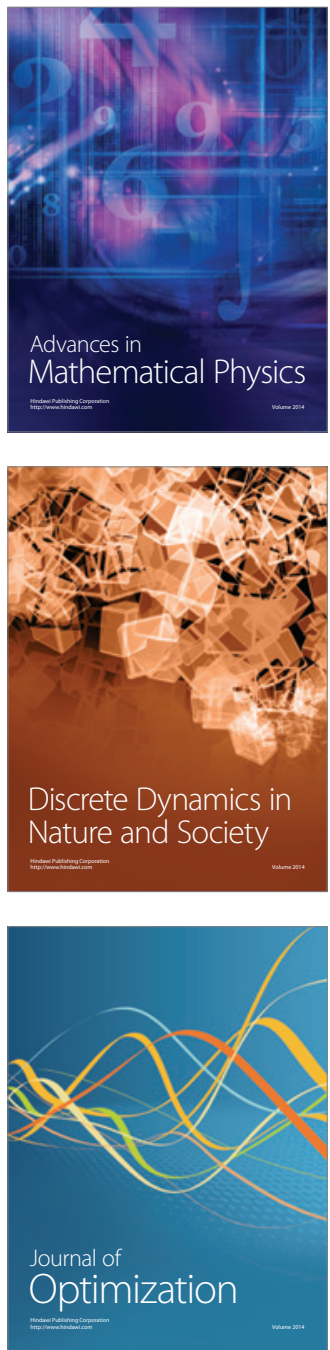